\title{
Adaptação e propriedades psicométricas da escala da paixão para docentes
}

http://dx.doi.org/10.11606/1807-5509201900020227

\author{
Maria Eloiza Fiorese PRATES* \\ Lenamar Fiorese VIEIRA** \\ Ieda Parra Barbosa RINALDI**
}

\author{
*Departamento de \\ Educação Física, \\ Universidade \\ Tecnológica Federal \\ do Paraná, Campo \\ Mourão, PR, Brasil. \\ **Departamento de \\ Educação Física, \\ Universidade Estadual \\ de Maringá, Maringá, \\ PR, Brasil.
}

\section{Resumo}

Este artigo realiza o processo de adaptação e análise das propriedades psicométricas da versão portuguesa do instrumento Scale of Passion de Vallerand et al. ${ }^{1}$, para docentes do contexto paranaense, obtida a partir do método de tradução cross-cultural, com base na Teoria do Modelo Dualista da Paixão. Dividindo-se em três fases: (1) adaptação transcultural e validade de conteúdo $(\mathrm{N}=27)$; (2) confiabilidade e validade de constructo $(\mathrm{N}=441)$; (3) fidedignidade $(\mathrm{N}=87)$ aplicada a docentes de ensino médio e superior de diferentes áreas que lecionam nas regiões norte central e centro ocidental do estado do Paraná. Concluímos que a sua versão com 14 itens mostrou ser uma medida com qualidades psicométricas, indicando ser aplicável aos docentes do contexto paranaense, como um instrumento de avaliação da paixão pela atividade docente.

Palavras-Chave: Validação; Paixão; Professor; Ensino.

\section{Introdução}

O rigor científico presente nas pesquisas tem levado a crescente avanço dos pesquisadores em busca de instrumentos de avaliação. Grande parte, porém, dos instrumentos psicométricos é decorrente de pesquisas desenvolvidas pelos pesquisadores de outros países, especificamente na área de psicologia, educação física e da educação. Para tanto, faz-se necessária à adaptação desses instrumentos da cultura do país de origem para a cultura brasileira ${ }^{2}$.

Entre as temáticas desenvolvidas em estudos nessas áreas, voltadas à profissão professor, estão as que utilizam questionários que envolvem a investigação do exercício profissional e da atividade docente, especialmente nos aspectos voltados a motivação $0^{3,4}$, a carreira e trabalho docente ${ }^{5-7}$, ao nível de qualidade de vida ${ }^{8}$ e atratividade da carreira docente?

No estudo realizado pela Fundação Victor Civita/ Fundação Carlos Chagas sobre a "Atratividade da Carreira Docente no Brasil", apenas 2\% dos estudantes de ensino médio, pretendiam realizar o curso de Pedagogia ou Licenciatura ${ }^{9}$. No período 2012-2013, de acordo com o resumo técnico do Censo de Educação superior de 2012, enquanto que nos cursos de bacharelado a matrícula foi de $67,5 \%$, os cursos de licenciatura e tecnologia tiveram apenas $18,9 \%$ e $13,7 \%$, respectivamente. Ainda se observa, nesse documento, que no período de 2009 a 2012, as matrículas em cursos de licenciatura, aumentaram pouco mais de $4 \%$ ao ano ${ }^{10}$. Sendo assim, nos levando a um quadro de $45,39 \%$ de vagas ociosas na formação de professores.

Diante desse cenário, observa-se que há uma visão negativa da sociedade em relação à carreira docente, ofuscando o lado positivo do profissional formado na área. Portanto, entender o que torna a vida dos professores mais gratificante pode dar sentido na profissão, mudando o foco dos fatores negativos para as qualidades positivas. Trata-se de buscar traços individuais positivos dos professores, como capacidade, vocação, habilidade, sensibilidade, perseverança, talento e sabedoria ${ }^{11}$. 
Assim, a carreira profissional do professor é resultado de fatores motivacionais, que se combinam e interagem de diferentes formas, que envolvem essa profissão e são amplamente discutidos na Teoria da Auto-determinaçáo de Ryan e Deci ${ }^{12}$. Desta abordagem se originou o Modelo Dualista da Paixão (MDP), propondo que as atividades agradáveis sáo internalizadas na medida em que são altamente valorizadas pelas pessoas, quando essa internalização ocorre dentro da identidade de alguém, leva a paixão pela atividade ${ }^{1}$.

Além disso, o MDP propóe que existem dois tipos de paixão, a obsessiva ( $\mathrm{PO}$ ) e a harmoniosa $(\mathrm{PH})$, que podem ser distinguidas em termos de como a atividade passional foi internalizada na identidade da pessoa. A paixão pela atividade pode ser harmoniosa, quando se refere a um forte desejo de dedicar-se livremente à atividade que se ama, ou pode ser obsessiva, quando representa um desejo incontrolável de se exercer a atividade ${ }^{13}$. A grande diferença é que, ao contrário da obsessiva, no desenvolvimento harmonioso as pessoas não sentem um desejo incontrolável sobre a atividade passional, mas optam por fazêla livremente, participando da atividade pela qual são apaixonadas de forma consciente e segura. $\mathrm{O}$ que as atividades passionais têm em comum é a paixáo pelo que se faz, bem como a disposiçáo de tempo e de energia para a realizaçáa ${ }^{14,15}$. Finalmente, nesse modelo ainda existe uma terceira sub-escala, o Critério da Paixão (CP), que avalia os critérios da paixão na atividade, grau de envolvimento, amor pela atividade, bem como a percepção dos participantes em ver a sua atividade como uma paixão.

\section{Método}

Este estudo metodológico buscou realizar a adaptação transcultural do instrumento Scale of Passion, utilizando-se das recomendaçóes de Vallerand $^{21}$. Para análise das propriedades psicométricas do instrumento foi realizada a tradução e retrotradução de uma versão original em inglês para a versão em português do Brasil, sua validade de conteúdo e de face, bem como a validade de constructo e a confiabilidade mediante a Análise Fatorial Confirmatória e alfa de Cronbach.

A autorização para realização do processo de tradução e validação para a língua portuguesa, Brasil, foi concedida pelo professor Robert J. Vallerand, primeiro autor do instrumento Scale of Passion. A pesquisa foi aprovada pelo Comitê Permanente de Ética em Pesquisa
No cenário internacional, validações têm sido realizadas, inicialmente o artigo original no francês, por VALLERAND et al. ${ }^{1}$, posteriormente em inglês e francês por Marsh et al. ${ }^{16}$, em italiana o Zito e CoLombo $^{17}$ com 14 itens. Mais recentemente a escala foi validada para o contexto chinês por ZHAO et al. ${ }^{18}$ e também para o espanhol com Chamarro et al. ${ }^{19}$, com 17 itens.

Para língua portuguesa foi validado por GONÇALVES et al.$^{20}$ para o contexto de trabalhadores de Portugal. No Brasil, esse instrumento revisado ainda não tem sido utilizado por pesquisadores, uma vez que sua tradução e validação para o português ainda não foi realizada. Diante desse contexto, o objetivo deste estudo foi realizar o processo de tradução, adaptação e validaçáo dos itens quanto à clareza, pertinência e dimensão teórica por juízes; a validade fatorial confirmatória, e a consistência interna teste-reteste da versão portuguesa do instrumento Escala da Paixão (Scale of Passion).

Para realização desta investigação consideramos necessária a implementação de três fases complementares para o processo de validação do questionário Scale of Passion: na fase (1) cumprimos os procedimentos referentes à adaptação transcultural e validade de conteúdo do instrumento; durante a fase (2) realizamos os métodos adequados para avaliar a confiabilidade e a validade de constructo dos itens do instrumento; por fim, na fase (3), verificamos a fidedignidade do instrumento Scale of Passion por meio dos índices de estabilidade temporal do teste.

com Seres Humanos da Universidade Estadual de Maringá (UEM), por meio do Parecer nº 352.361.

\section{Instrumento original}

A Scale of Passion de Vallerand et al. ${ }^{1}$, é composta por 14 itens na sua primeira versão, nas línguas francesa e inglesa ${ }^{16}$, italiana ${ }^{17}$ e portuguesa ${ }^{20}$.

A versão mais recente é composta por 17 itens (QUADRO 1) que avalia dois tipos de paixão: paixão obsessiva (PO) e paixão harmoniosa $(\mathrm{PH})$, sendo incluído o critério da paixão $(\mathrm{CP})$. Essa versão foi validada para língua espanhola por Chamarro et al. ${ }^{19}$, e na versão chinesa por ZHAO et al. ${ }^{18}$, sendo que mantiveram os 17 itens. 
A escala do tipo "likert" de sete pontos, que variam de "não concordo em nada" (1) a "concordo muito fortemente" (7). Os resultados são atribuídos a cada subescala, obtidos a partir da média aritmética das respostas dadas aos itens correspondentes a cada subescala da paixão.

QUADRO 1 - Dimensões teóricas e respectivos itens da Scale of Passion (VAllerand et al. ${ }^{1}$, ZhaO et al. ${ }^{18}$ e ChAmarro et al. ${ }^{19}$ )

\begin{tabular}{|l|l|l|}
\hline Dimensáo & Itens & Descriçáo \\
\hline Paixão Obsessiva $(\mathrm{PO})$ & $2,4,7,9,11,12$ & $\begin{array}{l}\text { Representa um desejo incontrolável de } \\
\text { exercer a atividade. }\end{array}$ \\
\hline Paixão Harmoniosa (PH) & $1,3,5,6,8,10$ & $\begin{array}{l}\text { Representa um forte desejo de dedicar-se } \\
\text { livremente à atividade que se ama. }\end{array}$ \\
\hline Critério da Paixão $(\mathrm{CP})$ & 13 a 17 & $\begin{array}{l}\text { Para identificar se a paixão está presente no } \\
\text { indivíduo. }\end{array}$ \\
\hline
\end{tabular}

Fase 1 - Adaptaçáo transcultural e validade de conteúdo do instrumento Scale of Passion

Para estabelecer uma versão preliminar do instrumento foi utilizado o método da tradução reversa (backtranslation); de acordo com VAllerand $^{21}$, esta fase é muito importante no processo de validação transcultural, que envolveram 4 tradutores. Primeiramente, foram enviados a dois tradutores (de língua materna portuguesa) de forma independente, para traduzir para o português a Scale of Passion. Na sequência, as duas versôes na língua portuguesa foram encaminhadas aos dois outros tradutores (de língua materna inglesa) para traduzirem, novamente, para o inglês. Após a realização da tradução reversa, o próximo passo foi o julgamento pelo comitê de especialistas para avaliar e modificar de forma minuciosa as versóes preliminares do instrumento ${ }^{21,22}$. Dessa forma, após análise e discussão a respeito da inconsistência encontrada das versões, em língua portuguesa, o comitê de tradutores e o pesquisador chegaram a um consenso e foi elaborada a redação da primeira versão do instrumento na língua portuguesa.

Assim, a análise teórica dos 17 itens da Escala da Paixão foi realizada através da validade de conteúdo por três professores universitários doutores e doutorandos, que participaram com o papel de juízes-avaliadores. A planilha de avaliação de conteúdo foi elaborada para esta fase do estudo e enviada a cada juiz separadamente, sendo avaliada a clareza de linguagem, a pertinência prática, a relevância teórica e a dimensão teórica dos itens da Escala da Paixão, com a escala likert (5 pontos); também foi incluído um campo de observação para cada item para possíveis sugestóes e análises dos juízes.

O cálculo do Coeficiente de Validade do Conteúdo (CVC) foi realizado como proposto por Hernández-Nieto ${ }^{23}$ e com base na nota dos juízes para cada item do instrumento Escala da Paixão. Foi adotado um CVC superior a 0,8 . Para avaliar a confiabilidade das medidas das dimensóes teóricas do instrumento, foi utilizado o calculado do índice de concordância entre avaliadores, o coeficiente Kappa ${ }^{24}$.

Após o processo de tradução e adaptação, um estudo piloto foi realizado com a versão final do instrumento. Para essa fase, foram utilizados 20 professores de diferentes instituições de ensino, que foram selecionados por conveniência, com o objetivo de avaliar a qualidade e a coerência da linguagem e do conteúdo das questôes do instrumento, de acordo com recomendaçóes de VAllerand ${ }^{21}$. Os professores participantes possuíam características diferenciadas (sexo, experiência profissional, nível de atuação e questóes demográficas). Todos os envolvidos nas 3 fases do presente estudo consentiram de forma voluntária e assinaram o Termo de Consentimento Livre e Esclarecido (TCLE).

\section{Fase 2 - Confiabilidade e validade de constructo do instrumento Scale of Passion}

Neste momento da investigação foi utilizada uma amostra de 441 professores vinculados a Instituições de Ensino públicas e privadas, sendo 210 do ensino médio e 231 do ensino superior das 
cidades de Campo Mourão, Londrina e Maringá do Estado do Paraná, Brasil.

Foram estabelecidos os seguintes critérios para seleção da amostra: os municípios compreenderam os mais populosos das mesorregióes geográficas Norte Central (Londrina e Maringá) e CentroOcidental Paranaense (Campo Mourão) e onde estavam localizados os Núcleos Regionais de Educação (NRE); as instituições de ensino superior definimos pelas maiores IES públicas e privadas em número de professores e de alunos matriculados localizadas nestes municípios. Em relação às instituições de ensino médio, o método de seleção foi por conveniência, sendo escolhidas pelas facilidades de acesso do pesquisador e as que continham maior número de professores de nível médio. Os sujeitos deveriam ser professores da instituição e lecionarem no ensino superior ou no ensino médio, sendo escolhidos pelo método de seleção de amostra não-aleatória por conveniência, pois os professores que aceitaram participar do estudo estavam presentes na instituição, no dia em que foi autorizada a coleta.

Após obter as autorizaçóes para a coleta de dados da pesquisa dos os chefes dos Núcleos Regionais de Educação do Paraná (NRE) e reitores das IES, acordou-se a data adequada para realização da pesquisa com os professores. A aplicação do questionário Escala da Paixão aconteceu no segundo semestre de 2013, na sala dos professores. Os dados obtidos foram analisados com o auxílio dos softwares SPSS versão $20.0 \mathrm{e}$ AMOS versão 20.0.

Foi realizada a análise descritiva dos dados (média, desvio padrão) para dados contínuos e frequência (percentual) para dados categóricos. Posteriormente, para verificar a consistência interna do instrumento utilizou-se a Confiabilidade Composta e o teste de alfa de Cronbach. Para complementar essa análise, foi calculada a Confiabilidade Composta a partir dos valores dos pesos fatoriais na sua forma estandardizada, resultantes da Análise Fatorial Confirmatória, esta medida indica que valores de $\mathrm{FC} \geq 0.7$ são considerados apropriados e abaixo de 0.7 possam ser aceitáveis ${ }^{25,26}$.

Para avaliar a qualidade de ajustamento do modelo Escala da Paixão foi utilizada Análise Fatorial Confirmatória (AFC) ${ }^{25}$. O número de fatores foi estabelecido de acordo com a teoria do Modelo Dualista da Paixão. A existência de outliers foi avaliada pela distância quadrada de Mahalonobis $\left(D^{2}\right)$ e a normalidade das variáveis foi avaliada pelos coeficientes de distribuição univariada de assimetria $(s k)$ e curtose $(k u)$ e distribuição multivariada.

Como sugerido por Fornell e LARCKeR ${ }^{27}$, a Validade Convergente das dimensões de cada escala do presente estudo pode ser avaliada por meio da Variância Extraída Média (VEM), e valores superiores a 0,40 foram considerados indicadores aceitáveis de validade convergente ${ }^{24}$. Para a avaliação da Validade Discriminante das escalas foram comparados os valores da Variância Extraída Média (VEM) de cada fator com as Correlaçôes Inter-constructo ao Quadrado associadas com cada fator da escala.

\section{Fase 3 - Análise da fidedignidade por meio da estabilidade temporal da escala da paixáo}

Essa fase do estudo foi composta por 87 professores de diferentes áreas de conhecimento do estado Paraná, participantes da fase 2 , sendo 46 do ensino médio e 41 do ensino superior. Os participantes responderam aos questionários, em dois momentos diferentes (teste-reteste), que foram aplicados com intervalo de 7-14 dias após a testagem inicial.

O método de avaliação da fidedignidade utilizado foi o teste-reteste ou estabilidade temporal; os professores foram avaliados em dois momentos distintos (inicial e retestagem) com intervalo de 7 a 14 dias entre as coletas de dados.

Para a coleta de dados, os participantes foram abordados individualmente na sala de professores de acordo com seu horário de atividade extraclasse. No segundo semestre letivo de 2013.

O coeficiente de correlação intraclasse (CCI) foi utilizado para indicar a confiabilidade, medindo a similaridade média dos escores dos sujeitos nas duas avaliaçóes (teste e reteste) ${ }^{28,29}$. Para manter a consistência ao descrever a força relativa de acordo com a estatística kappa, foram designados para essa fase do estudo os intervalos correspondentes, conforme Landis e $\mathrm{KoCH}^{30}$, com os seguintes pontos de corte: (abaixo de $0=$ pobre; 0 a $0,20=$ fraca; 0,21 a $0,40=$ provável; 0,41 a $0,60=$ moderada; 0,61 a $0,80=$ substancial; e 0,81 a $1,00=$ quase perfeita). $\mathrm{Na}$ avaliação da fidedignidade foi adotado o coeficiente de correlação intraclasses do teste-reteste os quais aceitam valores entre 0,7 e $1,0^{26}$. 


\section{Resultados}

Os índices alcançados do CVC, na fase 1 do estudo, para todos os itens de cada dimensão foram todos superiores a 0,8 , indicando serem altamente aceitáveis quanto aos aspectos de clareza de linguagem, pertinência prática e relevância teórica. No que se refere ao cálculo do coeficiente Kappa, a média dos três juízes concordando com a dimensão original da Escala da Paixão de VAllerand et al. ${ }^{1}$ foi de 0,77 , valor considerado substancial ${ }^{22}$.
Os resultados alcançados do CVC e de confiabilidade das medidas indicam que a versão traduzida e adaptada da Escala da Paixão apresenta uma linguagem clara para o português, além de ser relevante e pertinente aos professores do contexto paranaense.

As características sócio demográficas do grupo participante nessa fase estão distribuídas na TABELA 1.

TABELA 1 - Caracterização sociodemográfica dos grupos pesquisados

\begin{tabular}{|c|c|c|c|c|c|c|c|}
\hline \multirow{2}{*}{ Variável } & \multirow{2}{*}{ Categoria } & \multicolumn{2}{|c|}{ Ensino Médio } & \multicolumn{2}{|c|}{ Ensino Superior } & \multicolumn{2}{|c|}{ Total } \\
\hline & & f & $\%$ & f & $\%$ & f & $\%$ \\
\hline \multirow[t]{2}{*}{ Sexo } & Masculino & 68 & 71,4 & 114 & 49,4 & 182 & 41,3 \\
\hline & Feminino & 112 & 28,6 & 117 & 50,6 & 229 & 58,7 \\
\hline \multirow[t]{4}{*}{ Faixa etária } & $20-30$ & 33 & 15,7 & 46 & 19,9 & 79 & 17,9 \\
\hline & $31-40$ & 56 & 26,7 & 90 & 39,0 & 146 & 33,1 \\
\hline & $41-50$ & 74 & 35,2 & 63 & 27,3 & 137 & 31,1 \\
\hline & 51 acima & 47 & 22,4 & 32 & 13,9 & 79 & 17,9 \\
\hline \multirow[t]{3}{*}{ Estado civil } & Solteiro & 54 & 25,7 & 58 & 25,1 & 112 & 25,4 \\
\hline & Casado & 121 & 57,6 & 150 & 64,9 & 271 & 61,5 \\
\hline & Outros & 05 & 16,7 & 23 & 10,0 & 58 & 13,2 \\
\hline \multirow[t]{4}{*}{ Nível de atuação } & Médio & 198 & 94,3 & 02 & 0,9 & 200 & 45,4 \\
\hline & Médio/Graduação & 12 & 5,7 & 20 & 8,6 & 32 & 7,3 \\
\hline & Graduação & - & - & 114 & 49,4 & 114 & 25,9 \\
\hline & Graduação/Pós & - & - & 95 & 41,1 & 95 & 21,5 \\
\hline \multirow{4}{*}{$\begin{array}{l}\text { Ciclo de } \\
\text { desenvolvimento } \\
\text { profissional }^{28}\end{array}$} & Entrada & 35 & 16,7 & 46 & 19,9 & 81 & 18,4 \\
\hline & Consolidação & 35 & 16,7 & 48 & 20,8 & 83 & 18,8 \\
\hline & Diversificação & 79 & 37,6 & 98 & 42,4 & 177 & 40,1 \\
\hline & Estabilização & 61 & 29,0 & 39 & 16,9 & 100 & 22,7 \\
\hline \multirow{3}{*}{$\begin{array}{l}\text { Local de trabalho/ } \\
\text { instituiçáo }\end{array}$} & Pública & 171 & 81,4 & 124 & 53,7 & 295 & 66,9 \\
\hline & Privada & 07 & 3,3 & 90 & 39,0 & 97 & 22,0 \\
\hline & Pública/Privada & 32 & 15,2 & 17 & 7,4 & 49 & 11,1 \\
\hline
\end{tabular}

Esses resultados sugerem que os grupos pesquisados são compostos, predominantemente, de professores entre 31 e 50 anos, casados, que se encontram no ciclo de diversificaçãa ${ }^{31}$ ( 7 a 19 anos) e lecionam em instituiçóes de ensino públicas para estudantes de ensino médio, graduação e pós-graduação.

As médias das respostas de cada dimensão situaram-se entre a menor média de 2,48 para o item 2 ("Eu tenho dificuldades para controlar meus impulsos de fazer essa minha atividade") da paixão obsessiva $(\mathrm{PO})$, tanto para os professores do ensino superior como os do médio. Mas, em relação às maiores médias houve diferença entre os valores e itens dos professores de ensino superior e médio, porém situadas na mesma dimensão o critério da paixáo $(\mathrm{CP})$, como segue: no ensino superior o item 14 ("Eu gosto desta atividade") obteve a média mais alta $(6,25)$; no ensino médio o item 15 ("Esta atividade é importante para mim") alcançou a média mais alta $(5,84)$. 


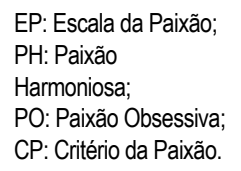

TABELA 2 - Consistência interna das dimensões da Escala da Paixão e correlação item- dimensão para professores do ensino superior e médio

\begin{tabular}{lcccc}
\hline \multicolumn{1}{c}{ Populaçáo } & Dimensóes (EP) & Item & $\alpha$ & Correlaçáo Item-dimensáo \\
\hline Professores do Ensino & PH & $1,3,5,6,8,10$ & 0,78 & $0,69 / 0,65 / 0,65 / 0,62 / 0,76 / 0,76$ \\
Superior & PO & $2,4,7,9,11,12$ & 0,81 & $0,54 / 0,71 / 0,73 / 0,72 / 0,82 / 0,75$ \\
& CP & $13,14,15,16,17$ & 0,78 & $0,55 / 0,72 / 0,79 / 0,85 / 0,83$ \\
\hline Professores do Ensino & PH & $1,3,5,6,8,10$ & 0,90 & $0,75 / 0,79 / 0,75 / 0,73 / 0,81 / 0,82$ \\
Médio & PO & $2,4,7,9,11,12$ & 0,83 & $0,49 / 0,73 / 0,72 / 0,72 / 0,80 / 0,64$ \\
& CP & $13,14,15,16,17$ & 0,83 & $0,49 / 0,85 / 0,85 / 0,87 / 0,87$ \\
\hline
\end{tabular}

O índice de consistência interna geral da Escala da Paixão para professores de Ensino Superior foi 0,83 e para professores de Ensino Médio foi 0,88, considerando-se o valor obtido aceitável e elevado, pois o limite mínimo aceito como satisfatório pela literatura é de $0,70^{25}$. A TABELA 2 apresenta a consistência interna das três dimensões do questionário e a correlação item-dimensão, bem como se pode observar que o alpha de Cronbach para cada dimensão foi satisfatório desde $\alpha=0,78$ a $\alpha=0,90$.

As correlaçóes item - escala variou de $0,54 \leq \mathrm{r} \leq 0,85$ (professores do ensino superior) e de $0,49 \leq \mathrm{r} \leq 0,87$ (professores do ensino médio), indicando moderada a forte correlação entre as dimensões e seus itens. Os pesos fatoriais (correlações entre os itens individuais e suas dimensões) são considerados significativos quando o valor for igual ou superior a $0,50(P F \geq 0,50)$, apresentando somente o item 2 da paixáo obsessiva e o item 13 do critério da paixão, abaixo desse índice (TABELA 2).

Nenhuma variável apresentou valores de $\mathrm{Sk}$ e Ku indicadores de violaçóes severas à distribuição normal $([\mathrm{SK}]<3[\mathrm{KU}]<10)^{25}$. Três observaçóes de professores de ensino médio e três do ensino superior apresentaram valores de $D^{2}$ que sugeriam que essas observaçóes eram outliers, logo, foi conveniente que a análise fatorial confirmatória fosse feita sem essas observaçōes.

O Modelo Escala da Paixão (MDP) submetido à $\mathrm{AFC}$ não apresentou diferenças em relação à extensão da versão original do instrumento com três dimensões. Assim, o modelo analisado pela
AFC postula a existência de três fatores latentes ou dimensões (paixão obsessiva, paixão harmoniosa e critério da paixão).

O modelo tri-fatorial da Escala da Paixão, com a versão traduzida original, ajustado com uma amostra de 210 professores do ensino médio e 231 do ensino superior de instituiçóes públicas e privadas dos municípios de Maringá, Londrina e Campo Mourão revelou uma qualidade de ajustamento inicial sofrível, como demonstrado na TABELA 3.

Para elaboração do modelo modificado, para professores de ensino médio e superior (FIGURA 1), três observações outliers foram eliminadas. Os itens 1, 2 e 13, cujos índices de modificaçóes sugeriam a saturação em dimensóes diferentes daqueles sugeridos na versão original da Escala da Paixão foram removidos e, por último, foram correlacionados os erros de medida para os professores de ensino médio dos itens 11 e 12 (PO), 8 e $10(\mathrm{PH})$, 14,16 e 17 (CP), e para os professores de ensino superior foram correlacionados os itens $7,9,11$ e 12 (PO), 8 e 10 (PH), 14, 16 e 17 (CP). Dessa forma, o modelo modificado apresentou uma qualidade de ajustamento significativamente superior à do modelo original na amostra, em suporte da validade fatorial da escala da paixão, para os professores que lecionam nos dois níveis de ensino (TABELA 3).

$\mathrm{Na}$ TABELA 3 foi realizada a comparação dos índices de qualidade dos modelos iniciais, após as alteraçôes efetuadas na estrutura do modelo. 
TABELA 3 - Índices de ajustamento do modelo Escala da Paixão para a amostra de validação dos professores de ensino médio e superior

\begin{tabular}{|c|c|c|c|}
\hline População & $\begin{array}{c}\text { Comparaçáo entre os } \\
\text { modelos da Escala da } \\
\text { Paixáo }\end{array}$ & Modelo com 17 itens & $\begin{array}{l}\text { Modelo modificado } \\
\text { com } 14 \text { itens* }\end{array}$ \\
\hline \multirow{12}{*}{$\begin{array}{l}\text { Professores de Ensino } \\
\text { Médio }\end{array}$} & $\mathrm{X}^{2}$ & 311,698 & 139,013 \\
\hline & $\mathrm{Gl}$ & 116 & 70 \\
\hline & $\mathrm{p}$-value & 0,000 & 0,033 \\
\hline & $\mathrm{X}^{2}$ normalized $\left(\mathrm{X}^{2} / \mathrm{df}\right)$ & 2,69 & 1,97 \\
\hline & GFI & 0,84 & 0,91 \\
\hline & RMSEA & 0,09 & 0,069 \\
\hline & TLI & 0,87 & 0,94 \\
\hline & AGFI & 0,79 & 0,87 \\
\hline & CFI & 0,89 & 0,96 \\
\hline & AIC & 1901,83 & 1710,27 \\
\hline & $\mathrm{BIC}$ & 1958,73 & 1756,92 \\
\hline & MECVI & 1,879 & 1,044 \\
\hline \multirow{12}{*}{$\begin{array}{l}\text { Professores de Ensino } \\
\text { Superior }\end{array}$} & $\mathrm{X}^{2}$ & 462,005 & 171,302 \\
\hline & $\mathrm{gl}$ & 116 & 69 \\
\hline & $\mathrm{p}$-value & 0,000 & 0.03 \\
\hline & $\mathrm{X}^{2}$ normalized $\left(\mathrm{X}^{2} / \mathrm{df}\right)$ & 4,37 & 2,48 \\
\hline & GFI & 0,79 & 0.91 \\
\hline & RMSEA & 0,121 & 0.08 \\
\hline & TLI & 0,74 & 0.90 \\
\hline & AGFI & 0,72 & 0,86 \\
\hline & CFI & 0.78 & 0.92 \\
\hline & AIC & 1746,93 & 1457,99 \\
\hline & $\mathrm{BIC}$ & 1805,45 & 1506,00 \\
\hline & MECVI & 2,25 & 1,09 \\
\hline
\end{tabular}

$\mathrm{X}^{2}$ : qui-quadrado; gl: Graus de liberdade; GFI: índice de qualidade do ajuste; RMSEA: raiz do erro quadrático médio de aproximação;

TLI: índice TuckerLewis;

AGFI: índice de qualidade de ajuste calibrado;

CFI: índice de ajuste comparativo;

AIC: critério de informação de Akaike; BIC: critério de informação Bayesiano; MECVI: índice de validação cruzada esperada;

*: Modelo adotado no estudo. 


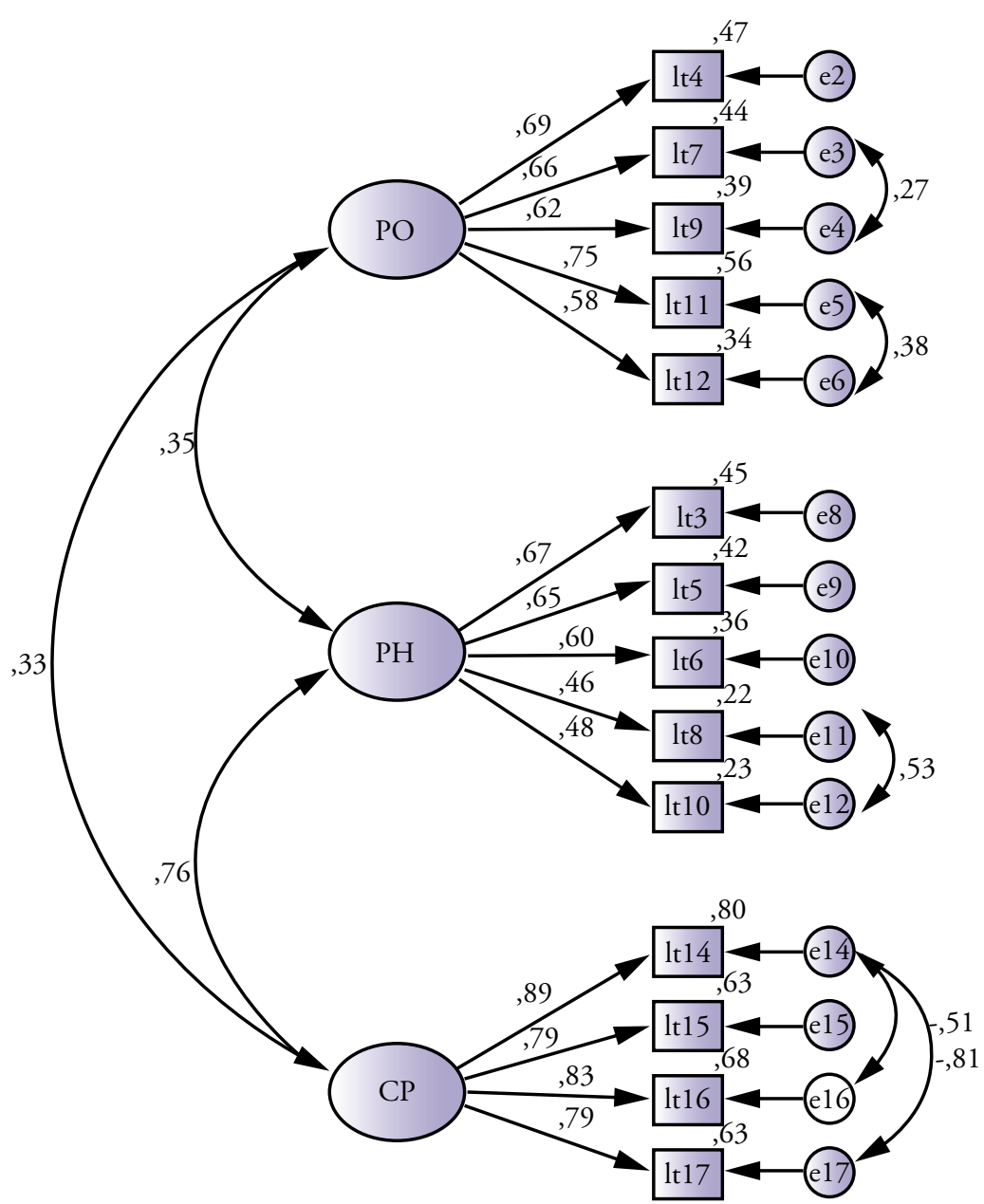

FIGURA 1 - Modelo tri-fatorial modificado da Escala da Paixão para professores.

Assim como os valores de AIC, BIC, MECVI e RMSEA em comparação ao modelo inicial apresentaram melhora, o modelo modificado apresentou uma qualidade de ajustamento significativamente superior à do modelo original na amostra, evidenciando um bom ajuste para professores do contexto paranaense, sugerindo a satisfatória dimensionalidade do instrumento para a língua portuguesa (Brasil). A FIGURA 1 apresenta o modelo final da Escala da Paixáo.

A análise da fiabilidade compósita (FC) tanto para os professores do ensino médio (EM) como para os do ensino superior (ES) indicou que os valores foram elevados, sendo que para o Ensino Médio correspondem a 0,84 para "Paixão Harmoniosa" $(\mathrm{PH}), 0,78$ para a "Paixão Obsessiva" (PO) e
0,93 para o "Critério da Paixão" (CP). A validade convergente dessas dimensôes foi verificada por meio da variância extraída média (VEM), apresentando $0,51(\mathrm{PH}), 0,42(\mathrm{PO})$ e $0,77(\mathrm{CP})$.

Para os professores de ensino superior, os resultados da FC foram de 0,71 (PH), 0,79 (PO) e 0,90 (CP). A VEM teve 0,34 (PH), 0,44 (PO) e 0,69 (CP). A Figura 1 apresenta os valores dos pesos fatoriais estandardizados e a fiabilidade individual de cada um dos itens do modelo modificado. Todos os valores foram superiores a 0,40 sendo, portanto, considerados indicadores aceitáveis de validade convergente ${ }^{32}$.

A validade discriminante das dimensóes foi avaliada pela comparaçáo das VEM com os quadrados da correlação entre as dimensóes. Os dados revelaram que nos resultados, tanto para 
os professores do ensino médio $\left(\mathrm{VEM}_{\mathrm{CP}}=0,77\right.$; $\left.\mathrm{VEM}_{\mathrm{PO}}=0,42 \mathrm{e} \mathrm{VEM}_{\mathrm{PH}}=0,51\right)$ como para os professores do ensino superior $\left(\mathrm{VEM}_{\mathrm{CP}}=0,69\right.$; $\left.\mathrm{VEM}_{\mathrm{PO}}=0,44 \mathrm{e} \mathrm{VEM}_{\mathrm{PH}}=0,34\right)$, os quadrados das correlaçóes das dimensóes são consideravelmente

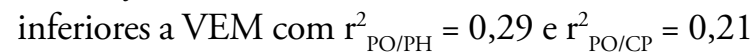
(ensino médio) $\mathrm{r}_{\mathrm{PO} / \mathrm{PH}}^{2}=0,12 \mathrm{e} \mathrm{r}^{2}{ }_{\mathrm{PO} / \mathrm{CP}}=0,11$ (ensino superior). Sendo assim, pode-se afirmar que essas dimensões têm validade discriminante.

No entanto, quando analisadas a $\mathrm{PH}$ com o $\mathrm{CP}$ o valor do quadrado da correlação entre as dimensóes tanto para os professores do ensino médio $\left(\mathrm{r}_{\mathrm{PH} / \mathrm{CP}}^{2}=0,77\right)$ quanto para os professores do ensino superior $\left(\mathrm{r}_{\mathrm{PH} / \mathrm{CP}}^{2}=0,58\right)$ foram superiores a VEM, não apresentando validade discriminante entre as mesmas. Contudo, apesar de não se ter verificado a validade discriminante entre todos os fatores na presente amostra, como proposto por MARÔCO ${ }^{25} \mathrm{com}$ valores superiores a $0.50 \mathrm{da}$ VEM, conceitualmente eles avaliam diferentes facetas da escala, por isso optou-se pela sua manutenção.

Dessa forma, a numeração e a ordenação dos itens (1 a 14), foram reorganizadas conforme o QUADRO 2 para as duas populaçôes estudadas, ocorrendo a eliminação de três itens do original (1, 2 e 13). Ficando assim ordenados.

QUADRO 2 - Dimensões da Escala da Paixão validada para professores do contexto paranaense

\begin{tabular}{|l|l|l|}
\hline Dimensão & $\begin{array}{l}\text { Itens para nova } \\
\text { versáo }\end{array}$ & Descrição \\
\hline Paixão Obsessiva $(\mathrm{PO})$ & $2,5,7,9$ e 10 & Representa um desejo incontrolável de exercer a atividade. \\
\hline Paixão Harmoniosa $(\mathrm{PH})$ & $1,3,4,6$ e 8 & $\begin{array}{l}\text { Representa um forte desejo de dedicar-se livremente à atividade } \\
\text { que se ama. }\end{array}$ \\
\hline Critério para Paixão $(\mathrm{CP})$ & $11,12,13$ e 14 & Para identificar se a paixão está presente no indivíduo. \\
\hline
\end{tabular}

A fidedignidade ou a precisão de um teste, analisada na fase 3 , diz respeito à característica que deve possuir, a de medir sem erros, significa que medindo os mesmos sujeitos em ocasiōes diferentes ou na mesma ocasião os resultados serão idênticos, isto é, a correlação entre as duas medidas deve ser 1 . No entanto, como sempre existem erros de medida, quer mostrar precisamente o quanto este se afasta do ideal da correlaçáo 1 , determinando um coeficiente que, quanto mais próximo de 1 , menor o erro que o teste comete ao ser utilizado ${ }^{2}$.

A medida de fidedignidade de estabilidade temporal do instrumento, de acordo com VALLERAND $^{21}$, é avaliada solicitando aos sujeitos que respondam ao instrumento duas vezes com um intervalo de tempo (cerca de 7 a 14 dias) entre as duas aplicaçóes do instrumento. Se o instrumento é fiel, as pontuaçóes dos sujeitos devem se manter estáveis. Índices de correlação de teste e reteste de aproximadamente 0,60 ou mais para esse período são satisfatórios.
Os resultados encontrados na fase 3 do coeficiente de correlaçáo intraclasse ( $r$ ), dos itens da Escala da Paixão, ficaram entre $r=0,44$ e $r=0,80$, com valor médio de 0,63 para professores de Ensino Médio e $r=0,47$ e $r=0,84$, com valor médio de 0,71 para professores de Ensino Superior, indicando valores moderado e quase-perfeita. Na maioria (86\%) dos professores do ensino superior e (57\%) do ensino médio participantes do estudo foram observadas correlaçóes substanciais, entre 0,81 e 1,00, conforme valores indicados por Landis e $\mathrm{KoCH}^{30}$.

Os resultados do coeficiente de correlação intraclasse (r) das três dimensões da Escala da Paixão: paixão harmoniosa, paixão obsessiva e critério da paixão, indicaram uma correlação quase perfeita na harmoniosa e critério da paixão, no entanto foi substancial para a paixão obsessiva, com os professores que atuam no ensino médio. Já para os professores do ensino superior houve uma correlaçáo quase perfeita na obsessiva e critério da paixão, sendo substancial na paixão harmoniosa (TABELA 4).

TABELA 4 - Coeficiente de correlação intraclasse (r) das três dimensões da Escala da Paixão

\begin{tabular}{lcccc}
\hline Populaçáo & Dimensóes & $\alpha$ (geral) & r & Intervalo de Confiança (95\%) \\
\hline \multirow{2}{*}{ Professores do } & PH (It 1, It 3, It 4, It 6 e It 8) & 0,90 & 0,81 & $0,69-0,89$ \\
Ensino Médio & PO (It 2, It 5, It 7, It9 e It 10) & 0,85 & 0,74 & $0,58-0,85$ \\
& CP (It 11, It 12, It 13, It 14) & 0,91 & 0,83 & $0,71-0,90$ \\
\hline
\end{tabular}

Continua 
Continuação

PH: Paixão Harmoniosa; PO: Paixão Obsessiva; CP: Critério da Paixão.

TABELA 4 - Coeficiente de correlação intraclasse (r) das três dimensões da Escala da Paixão

\begin{tabular}{llccc}
\hline Populaçáo & \multicolumn{1}{c}{ Dimensóes } & $\alpha$ (geral) & r & Intervalo de Confiança (95\%) \\
\hline \multirow{2}{*}{ Professores do } & PH (It 1, It 3, It 4, It 6 e It 8) & 0,87 & 0,74 & $0,56-0,85$ \\
Ensino Superior & PO (It 2, It 5, It 7, It9 e It 10) & 0,92 & 0,84 & $0,72-0,91$ \\
& CP (It 11, It 12, It 13, It 14) & 0,89 & 0,80 & $0,65-0,89$ \\
\hline
\end{tabular}

A correlação intraclasse foi confirmada com a análise do teste-reteste (estabilidade temporal) que indicou a confiabilidade da Escala da Paixão. A maioria das dimensôes da escala apresentou coeficiente acima do índice mínimo recomendado pela literatura $(r>0,75)$, sendo que também para VAllerand ${ }^{21}$, índices acima de 0,60 são considerados satisfatórios.

\section{Discussão}

A versão adaptada e traduzida da Escala da Paixão (fase 1) apresentou valores de validade de conteúdo semelhantes aos encontrados na literatura ${ }^{1,14}$. A estrutura final da Escala da Paixão manteve as dimensões da escala original ${ }^{1}$. Essa coerência com a escala original confirma o processo de validade de conteúdo da adaptação e tradução transcultural do instrumento.

A variabilidade dos professores do estudopiloto foi intencional para alcançar condiçóes mais semelhantes com os critérios sugeridos para testar o modelo em relação ao instrumento original e também permitir uma maior generalização ${ }^{25}$. Nossos resultados mostraram que a adaptação obtida é relevante para os professores do estudo piloto, corroborando com os resultados da análise de validade de conteúdo.

A validade do modelo teórico, testada na fase 2, define as relaçóes causais e hipotéticas, entre as variáveis independentes e dependentes ${ }^{25}$. Verificamos se a Scale of Passion representa com exatidão o conceito do Modelo Dualista da Paixão, no âmbito da Análise de Equaçóes Estruturais (AEE). Foi estimada a validade relacionada com o constructo, por meio da validade fatorial, validade convergente e discriminante.

A versão brasileira da Escala da Paixáo demonstrou indicadores satisfatórios de consistência interna e validade de construto, semelhante à validação espanhola de Chamarro et al. ${ }^{19}$ e chinesa de ZHAO et al. ${ }^{18}$ No entanto, para alcançar a qualidade de ajustamento do modelo latente foram necessárias algumas modificaçóes na estrutura fatorial, houve a exclusão de três itens (1, 2 e 13), sendo um de cada dimensão e também foram correlacionados alguns erros de medida. Sendo assim, a versão final ficou composta por 14 itens, com bons indicadores para o modelo para professores do contexto paranaense. O item 13 também apresentou cargas fatoriais baixas $(0,46)$ na validaçáo italiana, entretanto o item foi mantido sendo considerado um valor satisfatório ${ }^{17}$. Nas versóes espanhola e chinesa nenhum item foi excluído ${ }^{19,18}$.

O instrumento Escala da Paixão revelou escores de confiabilidade que apresentaram credibilidade de forma consistente e reprodutível das medidas, com índices de consistência interna superiores de 0,7 que é considerado um indicador de fiabilidade apropriado para investigaçóes exploratórias pela literatura ${ }^{32,25}$. Nesse caso, também foi possível verificar similaridade nos resultados dos cálculos de confiabilidade de cada dimensão da escala analisados separadamente (TABELA 2), confirmando que os itens são precisos nas suas medidas em relação a cada uma das dimensões de origem.

No estudo de validação da versão original do instrumento VALLERAND et al. ${ }^{1}$, a confiabilidade (alfa de Cronbach) dos dois fatores foi avaliada usando amostra $(n=520)$. Os resultados revelaram níveis aceitáveis de confiabilidade das subescalas tanto para a $\mathrm{PO}(=0,89)$ e $\mathrm{PH}(=0,79)$. A correlação entre as duas sub-escalas foi $0,46 \mathrm{e}$ as médias para as duas subescalas em todas as atividades foram 5,01 (SD = 1,15) e 3,20 (DP 1,47) para PH e PO, respectivamente. $\mathrm{Na}$ versão espanhola os valores médios (e desvio padrão) variaram de 4,10 a $5,30(1,49-1,89)$ para itens da $\mathrm{PH}$ e de 2,00 a 2,96 $(1,46-1,96)$ para itens da $\mathrm{PO}^{19}$. 
Os valores de correlação item-dimensão (TABELA 2) foram satisfatórios, fornecendo evidências de que os itens avaliam o que têm a intenção de medir em cada dimensão. Coeficientes de correlação item - dimensão acima de 0,70 são considerados como indicadores de uma estrutura bem definida, considerando-se que o fator explica pelo menos $50 \%$ da variância do item individual $^{32}$. Esses resultados são semelhantes ao estudo de Chamarro et al. ${ }^{19}$, no qual as relaçóes entre dimensões harmoniosas e obsessivas e itens de critério da paixáo replicaram razoavelmente os resultados de estudos anteriores. Ambos os escores apresentaram consistência interna adequada $(\alpha=0,81)$. Como também no estudo de ZHaO et al. ${ }^{18}$ que revelou a consistência interna aceitável de ambas as subescalas da Escala da Paixão chinesa. Especificamente, a confiabilidade foi de 0,86 para $\mathrm{PH}$ e 0,82 para PO.

Principais inconsistências da Escala da Paixão com a escala original foram verificadas com a Análise Fatorial Confirmatória (AFC). Três dos 17 itens tiveram cargas fatoriais abaixo de 0,50 . O item 13 mostrou carga fatorial menor que 0,30 , descrito como ponto de corte na literatura ${ }^{26}$. Todos os outros itens excluídos tiveram cargas fatoriais maiores que 0,40 o que é aceitável na literatura ${ }^{27}$. No entanto, esses mesmos itens mostraram alta correlação entre os erros de medidas, o que sugere uma variância e que afeta a identificaçáo do modelo latente.

Em nossos resultados, indicadores do modelo da Escala da Paixão eram pobres e não aceitáveis para a versão com 17 itens (TABELA 3), e os problemas foram evidenciados na análise de nível de item a item. No entanto, quando foram excluídos os itens problemáticos, o modelo com 14 itens foi considerado aceitável para os padróes da literatura $\mathrm{a}^{25}$. Os problemas se apresentaram semelhantes para a populaçáo de professores de ensino médio e superior. Contrariando o que foi encontrado nas versôes espanhola ${ }^{19}$ e chinesa ${ }^{18}$, onde as análises psicométricas demonstraram que em ambas as versōes mantiveram todos os itens das dimensōes $\mathrm{PH}, \mathrm{PO}$ e CP.

A medida de $D^{2}$, é necessária para validação de um modelo estrutural, a exclusão das análises de três observaçóes de professores foi realizada por apresentar valores extremos. De acordo com MARôCO ${ }^{25}$, a presença do registro dessas observações pode aumentar ou reduzir as covariâncias entre as variáveis. Sendo assim, foi importante suas exclusōes para melhorar a qualidade do modelo estrutural.
Os valores de $s k$ e $k u$ demonstraram não existir violação severa no modelo. Dessa forma, pode-se concluir que náo ocorreram desvios de normalidade capaz que comprometer a sua fiabilidade.

A partir dos resultados da Análise Fatorial Confirmatória foi possível verificar que o novo modelo apresenta índices de qualidade de ajustamento que revelam uma adequação boa da estrutura modificada da Escala da Paixáo à amostra do estudo (professores do ensino médio e superior). Assim, testamos culturalmente o modelo pré-validado da Escala da Paixáo para professores de ensino médio e superior. Destaca-se que o estudo de validação da china envolveu estudantes de mestrado, doutorado e graduação, evidenciando modelo satisfatório com índices de consistência interna estáveis ${ }^{18}$.

Para demonstrar a validade do constructo no âmbito da AEE, foi necessário apresentar os resultados de 3 componentes a validade fatorial (pesos fatoriais), convergente (calculada pela VEM) e discriminante (VEM e diferença do $X^{2}$ ). Embora nossos resultados da AFC tenham apresentado validade convergente e validade discriminante nas dimensóes do modelo, foram identificadas algumas correlaçóes entre os erros de alguns itens, indicando a existência de itens problemáticos.

Novos estudos poderão ser realizados com intuito de revisar esses erros de correlaçóes e, eventualmente, reescrever alguns desses itens para garantir uma estrutura fatorial mais clara. No entanto, se as correlaçóes entre os erros não afetam a identificação do modelo, elas podem ser admitidas e o modelo aceito, que é o caso de nosso estudo ${ }^{33,25}$.

Sendo assim, usando os valores das estatísticas do $X^{2}$ (qui-quadrado) e respectivos graus de liberdade dos modelos originais sem outliers e do modelo modificado, pode-se concluir que os modelos modificados se ajustam melhor à estrutura correlacional observada entre os itens tanto para os professores de nível de ensino médio, como também para os do ensino superior do que os modelos originais. Os índices incrementais de ajuste, CFI, GFI e TLI se apresentaram mais elevados, no modelo modificado (TABELA 2), indicando maior credibilidade e poder de explicaçáo comparada com o modelo original. E, os índices AIC, BIC e MECVI indicaram valores menores do que o modelo original evidenciando ser mais bem ajustado e de menor complexidade para a população em estudo.

Todos os resultados, encontrados na fase 3 , dos itens e dimensóes da Escala da Paixão se apresentaram 
satisfatoriamente correlacionados ao reteste e não foram observadas correlaçôes negativas entre os itens e as dimensōes ${ }^{23}$. A análise de confiabilidade por meio do coeficiente de correlação intraclasse mostrou que tanto os 14 itens como as três dimensóes da Escala da Paixão demonstraram valores confiáveis.

Os resultados de correlaçáo intraclasse $d a$ Escala da Paixão indicam a validade temporal do instrumento brasileiro para medir a paixão pela atividade, o que fortalece o processo de validação realizado neste estudo.

Sendo assim, este foi o primeiro estudo a realizar uma validação transcultural de uma escala da paixão para português do Brasil, como também a mostrar evidências psicométricas para a Escala da Paixão com uma amostra de professores de ensino médio e superior. A maioria das propriedades psicométricas previamente relatadas, utilizando a Escala da Paixão, foi realizada sobre desempenho individual ${ }^{34,35}$, sobre o esporte, com atletas e treinadores ${ }^{14,36,37}$, também relacionado ao bem-estar das pessoas ${ }^{38,39}$, em diferentes atividades físicas (ski, karatê, dançarinos e jogadores de vídeo game) na espanhola ${ }^{19}$, (trabalhadores) na italiana ${ }^{17}$ e por fim na chinesa ${ }^{18}$ (estudantes universitários).

A versão brasileira da Escala da Paixão demonstrou resultados satisfatórios em análise de conteúdo (fase 1), validade interna (fase 2) e validade temporal (fase 3), resultando em um instrumento válido e confiável. É importante mencionar, todavia, que a escala foi reduzida para 14 itens, em contraste com os 17 itens da Escala da Paixão original, tendo em conta a confiabilidade e ajuste de critérios individuais ${ }^{25}$.

O processo de validação e de análise das propriedades psicométricas de medida psicológica, como da Escala da Paixão, é contínuo, exigindo múltiplas abordagens metodológicas. ForNell e LARCKER ${ }^{27}$ salientam que a maneira mais simples para descrever a forma como o sistema de teste proposto está integrada aos métodos de teste atuais é delinear a teoria do modelo de equaçóes estruturais, determinando primeiramente se as medidas têm propriedades psicométricas satisfatórias, sendo a confiabilidade (validade convergente), variância média extraída e validade discriminante para cada variável não observada.

Um dos nossos principais resultados é a adequação do modelo de mensuração da escala com 14 itens. Apesar de ter menos itens, a estrutura interna é a mesma como nas escalas originais e com boa adequação para o modelo de fator de confirmação.

Outros estudos realizados com a Escala da Paixão também encontraram resultados satisfatórios testando seu modelo original ${ }^{1,16-20}$. Os autores optaram por adaptar o instrumento para aplicar na pesquisa sobre o jogo de azar, com a eliminação de 4 itens, sendo 2 de cada subescala (paixão obsessiva e harmoniosa), não utilizando, portanto, os itens relacionados ao critério da paixão, os resultados indicaram níveis adequados de confiabilidade e validade ${ }^{40}$. Os achados no estudo de Marsh et al. ${ }^{16}$ demonstraram que a Escala de Paixão tem uma boa estrutura fatorial, consistência interna e validade de constructo.

Os autores adicionaram ainda que o mesmo instrumento pode ser usado em pesquisas independentemente do sexo dos participantes, da língua (validação em francês e inglês) ${ }^{16}$ e do domínio da paixão pela atividade. Sendo assim, infere-se que a Escala de Paixão constitui uma nova medida que tem muito a contribuir para a avaliação psicológica em suas diferentes áreas ${ }^{1,16-20}$.

Uma limitaçáo do presente estudo está relacionada à distribuiçẫo geográfica da amostra. Todos os nossos professores foram do estado do Paraná. Além disso, todos os professores que participaram do estudo atuam no nível médio ou no superior. Estudos futuros devem abordar essas questóes relativas à validação de forma que os resultados possam ser generalizados para outras amostras independentes. Este estudo se comportou de forma semelhante à versão canadense, testada em 539 estudantes universitários ${ }^{1}$, e a realizada em chinês com 286 estudantes universitários ${ }^{18}$, apresentando boa qualidade de ajustamento, consistência interna e confiabilidade.

Nesse contexto, novos estudos podem ser realizados replicando as propriedades psicométricas do questionário da Escala da Paixão para outras amostras e outras culturas para confirmar a estabilidade dos resultados encontrados neste estudo, especificamente com abordagens confirmatórias. Além disso, outras propriedades psicométricas precisam ser abordadas, tais como parâmetros de validade externa, capacidade de resposta e item individual.

Como esta é a primeira versão do instrumento a ser validado para português do Brasil, é necessário que mais pesquisas sejam realizadas para estabelecer uma forte evidência da Escala da Paixão em relação à correlação da paixão com outras escalas já validadas como os estudos Vallerand et al. ${ }^{1}$, Marsh et al. ${ }^{16}$, Zito e Colombo ${ }^{17}$, Zhao et al. ${ }^{18}$, Chamarro et al. ${ }^{19}$ e Gonçalves et al. ${ }^{20}$

A Escala da Paixão em sua versão com 14 itens mostrou ser uma medida confiável e validada entre os professores do contexto paranaense, como um instrumento de avaliação da paixão pela atividade docente. 


\section{Abstract}

\section{Adaptation and properties psychometric scale of passion for teachers}

This article presents the process of adaptation and analysis of the psychometric properties of the Portuguese version of the Scale tool of Passion of Vallerand et al. ${ }^{1}$, for teachers of Paraná context, obtained from the cross-cultural translation method, based on the Dualist Model Theory of the Passion. Divided into three phases: (1) cross-cultural adaptation and content validity $(\mathrm{N}=7)$; (2) reliability and construct validity $(N=441)$; (3) reliability $(N=87)$ applied to high school teachers and higher from different fields who teach in the northeast, Parana State. We conclude that the version with 14 items proved to be a measure psychometric qualities, indicating that it applies to teachers of Paraná context as an assessment tool of passion for teaching activity.

KeYWORD: Validation; Passion; Teacher; Education.

\section{Referências}

1. Vallerand RJ, Blanchard CM, Mageau GA, et al. Les passions de l'âme: on obsessive and harmonious passion. J Pers Soc Psychol. 2003;85(4):756-76.

2. Pasquali L. Testes referentes a construto: teoria e modelo de construção. In: Pasquali L, organizador. Instrumentaçâo psicológica: fundamentos e práticas. Porto Alegre: Artmed; 2009. p. 165-98.

3. Carson RL, Chase MA. An examination of physical educations teacher motivations from a self-determination theoretical framework. Phys Educ Sport Pedagogy. 2009;14(4):335-53.

4. Minelli DS, Nascimento GY, Vieira LF, Barbosa-Rinaldi IP. O estilo motivacional de professores de educaçáo física. Motriz. 2010;16(3):598-609.

5. Carlotto MS. A síndrome de burnout e o trabalho docente. Psicol Estud. 2002;7(1):21-9.

6. Farias GO, Nascimento JV, Graça A, Batista PMF. Crenças e expectativas constituídas ao longo da carreira docente em educaçáo física. Rev Educ Fís UEM. 2011;22(4):497-509.

7. Farias GO, Nascimento JV. Fatores intervenientes na carreira de professores de educaçáo física. Pensar Prat. 2012;15(2):465-83.

8. Silva JVP, Nunez PRM. Qualidade de vida, perfil demográfico e profissional de professores de educação física. Pensar Prat. 2009;12(2):1-11.

9. Fundação Victor Civita. A atratividade na carreira docente no Brasil: relatório de pesquisa [Internet]. São Paulo: Fundação Vitor Civita; 2009 [citado 26 out 2011]. Disponível em: http://www.zerohora.com.br/pdf/15141177.pdf

10. Brasil. Ministério da Educação. Instituto Nacional de Estudos e Pesquisas. Resumo técnico do Censo da educação superior 2012 [Internet]. Brasília, DF: MEC; 2014 [citado 8 nov 2014]. Disponível em: http://portal.inep.gov.br/web/censo-daeducacao-superior/resumos-tecnicos

11. Seligman MEP, Csikszentmihalyi M. Positive psychology: an introduction. Am Psychol. 2000;55(1):5-14.

12. Ryan RM, Deci EL. Self-determination theory and the facilitation of intrinsic motivation, social development, and well-being. Am Psychol. 2000;55(1):68-78.

13. Lafrenière MAK, Jowett $S$, Vallerand RJ, Carbonneau N. Passion for coaching and the quality of the coach-athlete relationship: the mediating role of coaching behaviors. Psychol Sport Exerc. 2011;12(2):144-52.

14. Vallerand RJ, Salvy SJ, Mageau GA, et al. On the role of passion in performance. J Pers. 2007;75(3):505-34.

15. Vallerand RJ. The dualistic model of passion. In: Roberts GC, Treasure D, editors. Advances in motivation in sport and exercise. 3a ed. Champaign: Human Kinetics; 2012. p. 169-206.

16. Marsh HW, Vallerand RJ, Lafrenière MAK, et al. Passion: does one scale fit all? construct validity of two-factor passion scale and psychometric invariance over different activities and languages. Psychol Assess. 2013:25(3):796-809.

17. Zito M, Colombo L. The Italian version of the Passion for Work Scale: first psychometric evaluations. J Work Organ Psychol. 2017;33(1):47-53.

18. Zhao Y, St-Louis A, Vallerand RJ. On the validation of the Passion Scale in Chinese. Psych Well Being. 2015;5(3):1-11. 
19. Chamarro A, Penelo E, Fornieles A, Oberst U, Vallerand RJ, Fernández-Castro J. Psychometric properties of the Spanish version of the Passion Scale. Psicothema. 2015;27(4):402-9.

20. Gonçalves G, Orgambídez-Ramos A, Ferrão MC, Parreira T. Adaptation and initial validation of the passion scale in a Portuguese sample. Escritos Psicol. 2014;7(2):19-27.

21. Vallerand RJ. Vers une méthodologie de validation trans-culturelle de questionnaires psychologiques: implications pour la recherche en langue fraçaise. Can Psychol. 1989;30(4):662-80.

22. Cassepe-Borges V, Balbinotti MAA, Teodoro MLM. Tradução e validação de conteúdo: uma proposta para adaptação de instrumentos. In: Pasquali L, organizador. Instrumentação psicológica: fundamentos e práticas. Porto Alegre: Artmed; 2010. p. $506-20$.

23. Hernández-Nieto R. Contributions to statistical analysis: the coefficients of proportional variance, content validity and Kappa. Mérida: Universidad de Los Andes; 2002.

24. Baltar VT, Okano V. Análise de Concordância - Kappa [Internet]. Sáo Paulo: Laboratório de Epidemiologia e Estatística, Instituto Dante Pazzanese; 2005 [citado 9 out 2013]. Disponível em: http://lee.dante.br/pesquisa/

25. Marôco J. Análise de equaçôes estruturais: fundamentos teóricos, software e aplicaçôes. Pêro Pinheiro: ReportNumber; 2010.

26. Nunnally JC, Bernstein IH. Psychometric theory. New York: McGraw-Hill; 1994.

27. Fornell C, Larcker DF. Evaluation SEM with unobserved variables and measurement error. J Marketing Res. 1981;18(1):39-50.

28. Barbetta PA. Estatística aplicada às Ciências Sociais. 5a ed. Florianópolis: Editora da UFSC; 2002.

29. Barros MVG, Reis RS. Análise de dados em atividade física e saúde: demonstrando a utilização do SPSS. Londrina: Midiograf; 2003.

30. Landis JR, Koch GG. The measurement of observer agreement for categorical data. Biometrics. 1977;33(1):159-74.

31. Huberman M. O ciclo de vida profissional dos professores. In: Nóvoa A, organizador. Vidas de professores, volume 2. Porto: Porto Editora; 1992. p. 31-61.

32. Hair JF, Anderson RF, Tatham RL, Black WF, Babin BJ. Análise multivariada de dados. Porto Alegre: Bookman; 2005.

33. Blunch NJ. Introduction to structural equation modeling using IBM SPSS Statistics and AMOS. London: Sage; 2008.

34. Bélanger JJ, Lafrenière MA, Vallerand RJ, Kruglanski AW. Driven by fear: the role of failure information on passionate individuals' performance. J Pers Soc Psychol. 2013;104(1):180-95.

35. Lafrenière MAK, St-Louis AC, Vallerand RJ, Donahue EG. On the relation between performance and life satisfaction: the moderating role of passion. Self Identity. 2012;11(4):516-30.

36. Lafrenière MA, Jowett S, Vallerand RJ, Gonahue EG, Lorimer R. Passion in sport: on the quality of the coach-player relationship. J Sport Exerc Psychol. 2008;30(5):541-60.

37. Philippe FL, Vallerand RJ, Andrianarisoa J, Brunel P. Passion in referees: examining affective and cognitive experiences in sport situations. J Sport Exerc Psychol. 2009;31(1):77-96.

38. Philippe FL, Vallerand RJ, Lavigne G. Passion does make a difference in people's lives: a look at well-being in passionate and non-passionate individuals. Appl Psychol Health Well Being. 2009;1(1):3-22.

39. Rousseau FL, Vallerand RJ. An examination of the relationship between passion and subjective well-being in older adults. Int J Aging Hum Dev. 2008;66(3):195-211.

40. Rousseau FL, Vallerand RJ, Ratelle CF, Mageau GA, Provencher PJ. Passion and gambling: validation of the Gambling Passion Scale (GPS). J Gambl Stud. 2002;18(1):45-66.

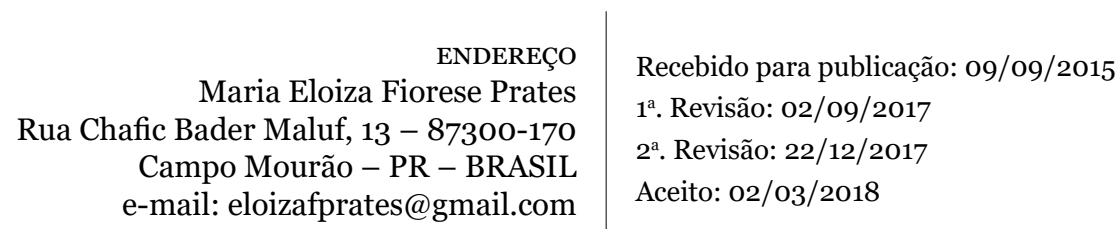

